\title{
Prediction of Electricity consumption for residential houses in New Zealand
}

\author{
Aziz Ahmad ${ }^{1}$, Timothy. N. Anderson ${ }^{2}$, and Saeed Ur Rehman ${ }^{2}$ \\ ${ }^{1}$ Unitec Institute of Technology, Auckland, 1142, New Zealand \\ ${ }^{2}$ Auckland University of Technology, Auckland, 1010, New Zealand \\ aahmad@unitec.ac.nz
}

\begin{abstract}
Residential consumer's demand of electricity is continuously growing, which leads to high greenhouse gas emissions. Detailed analysis of electricity consumption characteristics for residential buildings is needed to improve efficiency, availability and plan in advance for periods of high electricity demand. In this research work, we have proposed an artificial neural network based model, which predicts the energy consumption of a residential house in Auckland 24 hours in advance with more accuracy than the benchmark persistence approach. The effects of five weather variables on energy consumption was analyzed. Further, the model was experimented with three different training algorithms, the levenberg-marquadt (LM), bayesian regularization and scaled conjugate gradient and their effect on prediction accuracy was analyzed.
\end{abstract}

Keywords: Electricity demand forecast; load prediction; neural network; load management

\section{Introduction}

The residential sector of a country consumes $16-50 \%$ of energy of all sectors and averages approximately at $30 \%$ globally as shown in Figure 1 . The relatively high energy consumption of residential users demands a detail analysis of its energy consumption characteristics in order to promote conservation, efficiency, technological implementation and energy source switching, such as the utilization of on-site renewable energy sources [1].

Recent increase in the implementation of renewable energy systems has increased interest in the precise modeling and prediction of energy consumption. Energy production from renewable sources vary throughout 24 hours 365 days a year. Therefore, predicting the energy consumption 24 hour prior helps in efficient optimization of energy distribution among loads specifically between building and local grids. On the other hand, electricity consumption prediction is essential for generators, wholesalers and retailers of electric energy, who buy and sell, switch loads, plan maintenance and unit commitment and much more. However, with increasing costs passed to consumers, the optimum on-site energy generators can be achieved only when consumers can forecast their requirements in order to efficiently utilize grid-tied storage system and solar panels, thus enabling intelligent buildings. 
In [2], a building that minimizes the grid power usage and maximizes services efficiency is defined as an intelligent building. Sensors, renewable energy sources and energy management system (EMS) are some of the components of intelligent building with high ranking given to EMS, which monitors and controls the energy consumption. However, the effective operation of an EMS can be realized by precisely predicting the required power consumption. The early predication of load consumption would enable the planning of load distribution in advance and avoid power outages by shifting the unnecessary load to off peak time, to reduce cost and increase efficiency.

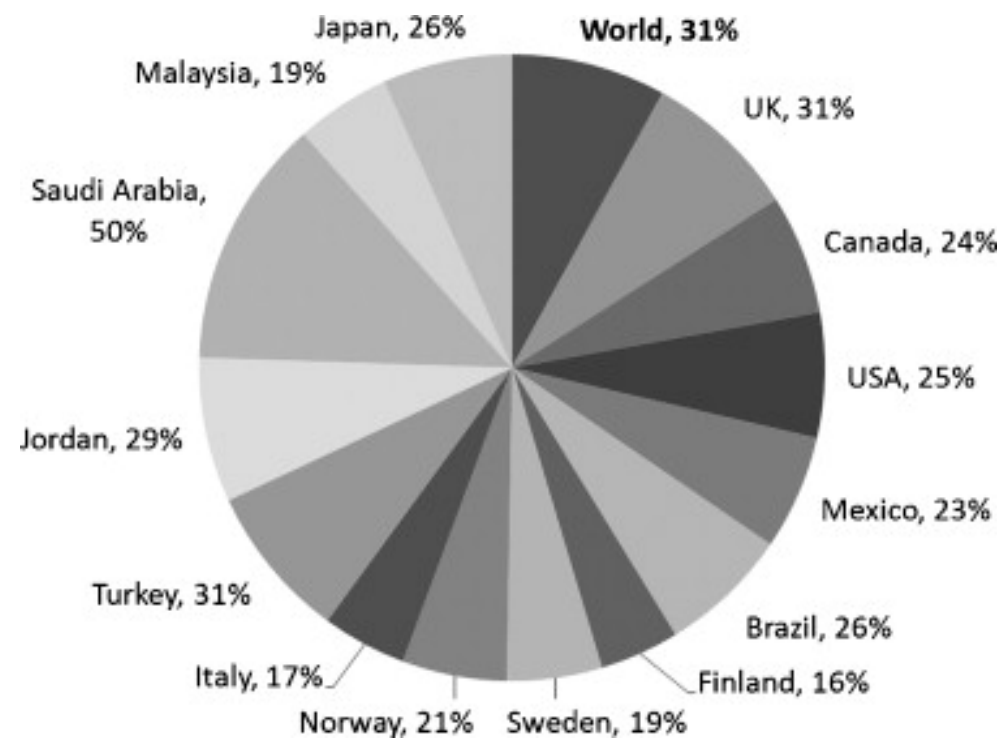

Figure 1. Worldwide energy consumption of residential users as a percentage of national energy usage of different sectors [1].

Short term load prediction of a residential house is a complex task due to the usage of various equipment with varying power requirement. Recently, short term load prediction has attracted considerable attention from researcher and scientists working both in academia and industry. A number of mathematical models and machine learning algorithms have been investigated for short term load prediction both in residential and small industry. Some of the algorithms used are regressive analysis, wavelet analysis, fuzzy system modeling, neural network modeling, evolutionary algorithms and hybrid approaches [3-10].

Artificial neural network (ANN) has been investigated for load forecasting mostly in commercial domain for large loads, such as ANN expert system [12-14]. ANN has the advantage to implicitly extract non-linear relationship among loading variables, learn and predict the future values. ANN has applications in different areas of power 
systems to handle complex non-linear functions, provide reliability and efficiency even for the cases where learning data is incomplete or not available at all $[14,15]$. In majority of previous work, ANN has been used for large scale forecasting. This research work uses different training algorithms of ANN for short-term load forecasting for a residential house.

Our main contribution of this research work is to train the ANN model with three different algorithms to predict electric load in a typical New Zealand residential house. A typical residential consumer would be living in a three bedroom house with four occupants ( 2 adults and 2 children). The goal is to predict the load 24 hours in advance, so that renewable energy sources and its components can be efficiently utilized. It is suggested that a similar model could be adopted for other locations with varying number of occupants.

\section{Methodology}

In this research work, an ANN based NARX (nonlinear autoregressive network with exogenous inputs) predictive model was used to forecast future values of electricity consumption. The NARX model uses historical values of electricity consumption and historical values of five environmental variables one of the input variable is hourly electricity data collected from a residential house in Auckland, New Zealand. The five environmental variables are: Temperature $\left(T_{\text {mean }}\right)$, Barometric Pressure $(P)$, Relative Humidity $(R H)$, Wind speed $\left(W_{s}\right)$ and Wind direction $\left(W_{d}\right)$. Data were taken from the National Institute of Water and Atmosphere's (NIWA) CliFlo database (2014), [19]. Our ANN model was trained on the historical electricity consumption and environmental variables data with electricity consumption 24 hours in advance being the output variable.

The predictive model can be expressed mathematically by predicting future values of the electricity consumption time series $\mathrm{y}(\mathrm{t})$ from past values of that time series and past values of input variables time series $\mathrm{x}(\mathrm{t})$ [16]. The equation for the NARX model is given by Equation 1

$$
\mathrm{y}(\mathrm{t})=f\left(\mathrm{y}(\mathrm{t}-1), \mathrm{y}(\mathrm{t}-2), \ldots, \mathrm{y}\left(\mathrm{t}-\mathrm{n}_{\mathrm{y}}\right), \mathrm{u}(\mathrm{t}-1), \mathrm{u}(\mathrm{t}-2), \ldots, \mathrm{u}\left(\mathrm{t}-\mathrm{n}_{\mathrm{u}}\right)\right)
$$

Where the next value of the dependent output signal y $(\mathrm{t})$ is regressed on previous values of the output signal and previous values of an independent input signal. The NARX model is implemented using a feed-forward neural network to approximate the function $\mathrm{f}$ in equation 1 . Figure 2 shows the diagram of the resulting network, where $y(t)$ output series is predicted given past values of $y(t)$ and another input series $x(t)$.

The prediction accuracy of ANN models is dependent on the combination of weather predictor variables and training algorithm [18].

Various combinations of weather predictor variables were tested using a statistical analysis to find the relationship between electricity consumption and individual weather variable as shown in Table 1 . The Pearson correlation coefficient $(R)$ is a measure of the linear correlation between two variables, giving a value between +1 and -1 inclusive, where 1 is total positive correlation, 0 is no correlation, and -1 is total negative correlation. 


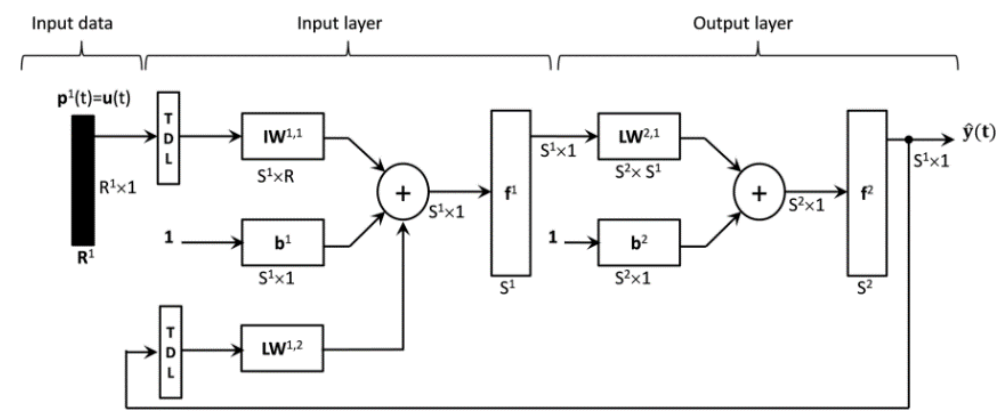

Figure 2. NARX network diagram

In order to determine the performance of the ANN models, the regression (R) and mean squared error (MSE) values were analyzed. The mean squared error (Equation 2) provides information on the short term performance and is a measure of the variation of predicated values around the measured data, lower MSE values represent better prediction accuracy.

$$
M S E=\frac{1}{N} \sum_{i=1}^{N}\left(I_{p, i}-I_{i}\right)^{2}
$$

Where the predicted electricity consumption in $\mathrm{kWh}$ is $I_{p}, I_{i}$ is the measured electricity consumption in $\mathrm{kWh}$, and $N$ denotes the number of observations.

Table 1. Regression (R) values for input weather variables vs electricity consumption

\begin{tabular}{ll}
\hline Input variables & $\mathrm{R}$ \\
\hline Temperature (Tmean) & 0.438 \\
Relative Humidity (RH) & 0.335 \\
Pressure (P) & 0.203 \\
Wind Speed (Ws) & 0.033 \\
Wind Direction (Wd) & 0.030 \\
\hline
\end{tabular}

Further, the models were experimented with three different training algorithms, the levenberg-marquadt, bayesian regularization and scaled conjugate gradient and their effect on prediction accuracy was analyzed as shown in Table 2.

Table 2. Effect of training algorithms on prediction accuracy

\begin{tabular}{llll}
\hline Input variables & MSE $(\mathrm{kWh})$ & $\mathrm{R}$ & Processing time \\
\hline Levenberg-Marquadt & 0.125 & 0.438 & $00: 00: 01$ \\
Bayesian regularization & 0.129 & 0.417 & $00: 01: 53$ \\
Scaled conjugate gradient & 0.142 & 0.360 & $00: 00: 02$ \\
\hline
\end{tabular}




\subsection{Electricity consumption prediction for a residential house}

Figure 3 and Figure 4 show four-days and one-day prediction of electricity consumption for a residential house in Auckland with 2 adults and two children respectively. It can be seen in Figure 3, Figure 4 and Table 2 that the LM training algorithms predicts electricity consumption more accurately than the BR and SCG training algorithms.

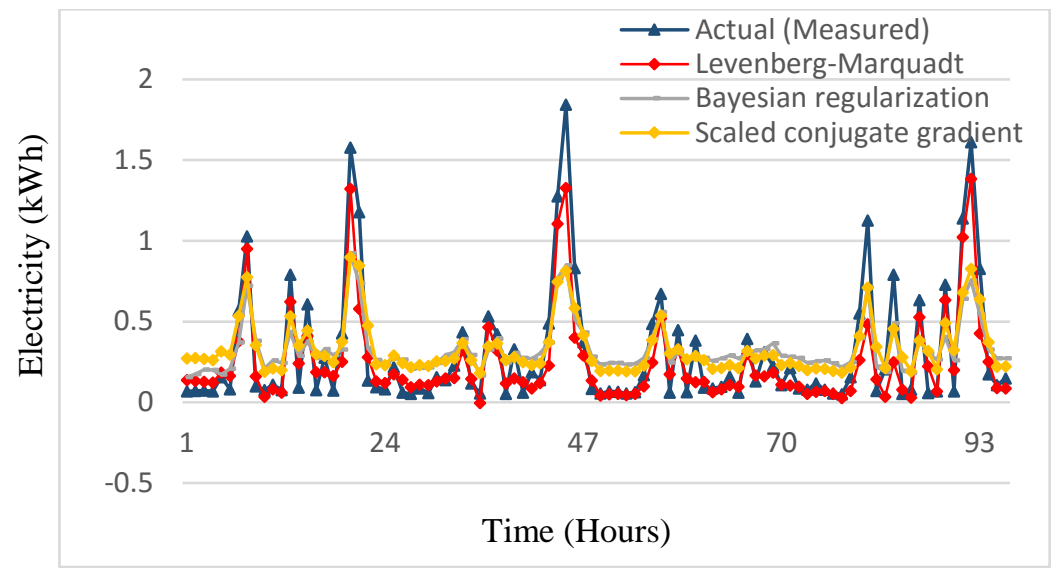

Figure 3. The predicted and measured values of electricity consumption for Four-days with MSE $=0.125 \mathrm{kWh}$

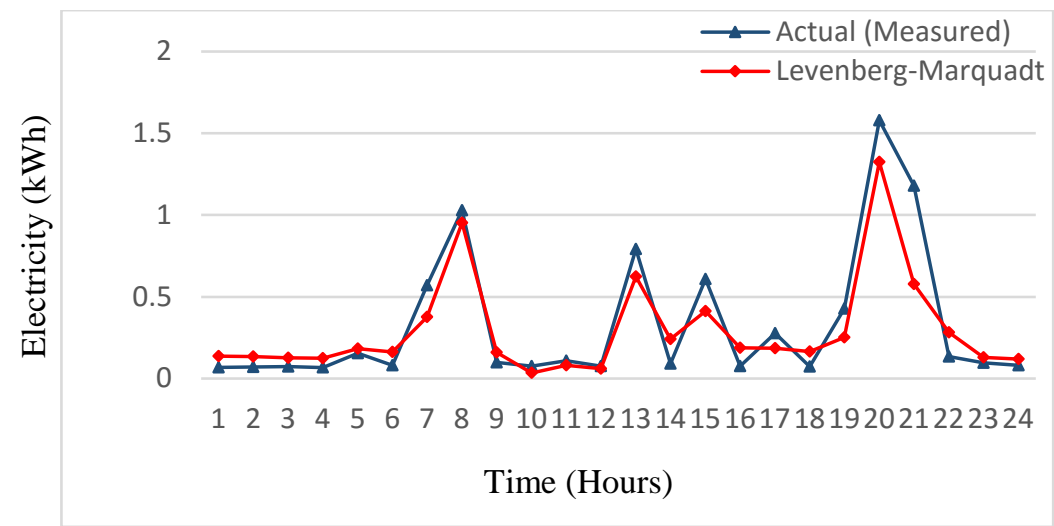

Figure 4. 24-hours measured and predicted values of electricity consumption for the LM algorithm

\subsection{A benchmark persistence model}

As a comparative study, the persistence model was developed using Equation 3 to predict the h hour-ahead forecasting ( $h=1,2,3 \ldots$ hours). 


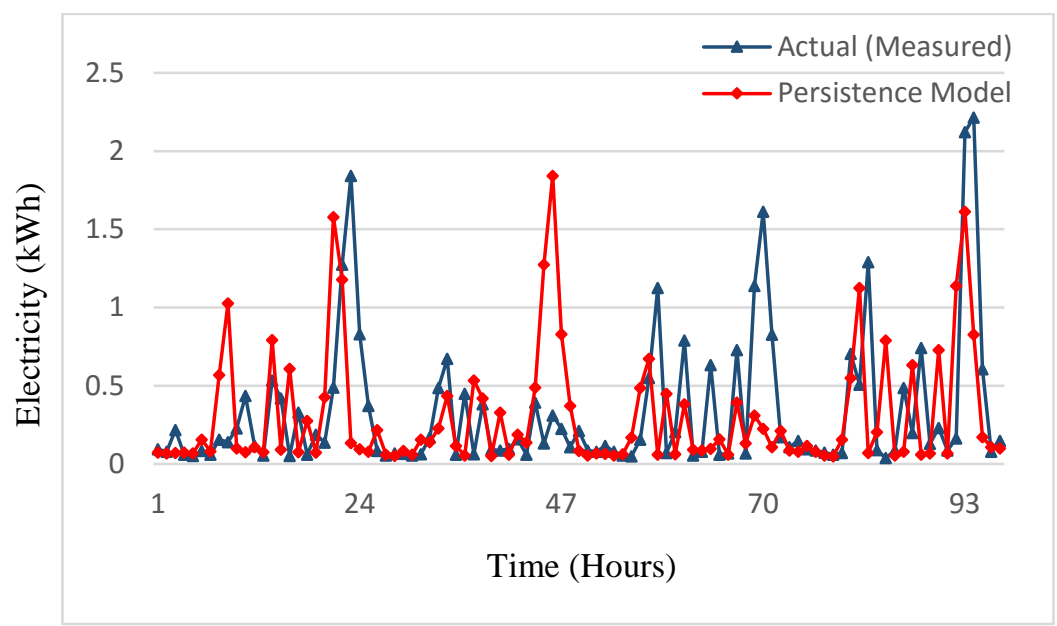

Figure 5. Four-days measured and predicted values of electricity consumption using the benchmark persistence approach with MSE $=0.998 \mathrm{kWh}$

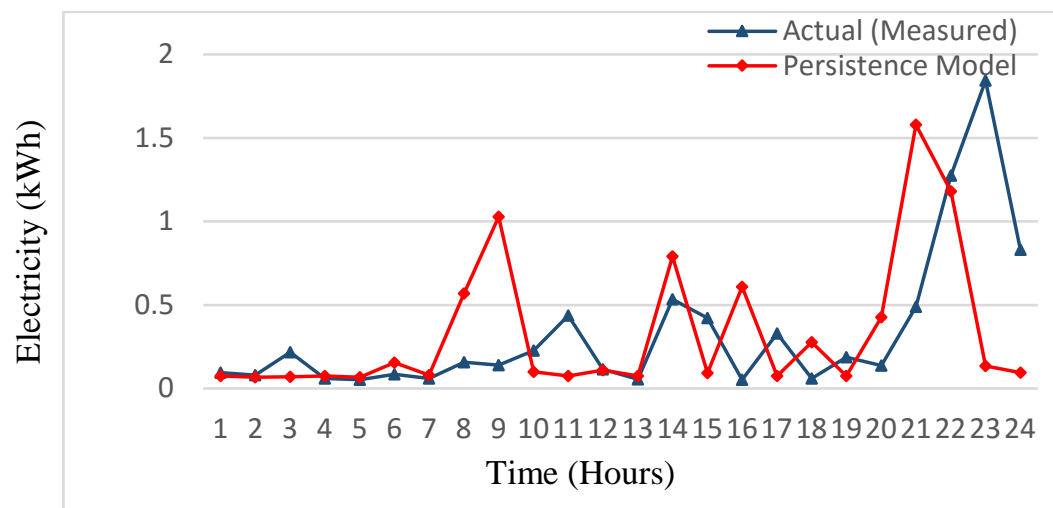

Figure 6. 24-hours measured and predicted values of electricity consumption using the benchmark persistence approach with MSE $=0.998 \mathrm{kWh}$

$$
S(t+h)=S(t)
$$

Where $S(t+h)$ is the predicted electricity consumption at time $t+h$.

Same electricity consumption and five input variables data used for the NARX model was utilized for the benchmark persistence approach, with electricity consumption as the objective function. Mean square error as defined in Equation 2 was calculated to validate and compare the model performance. Figure 5 and Figure 6 show four-days and one-day prediction of electricity consumption for a residential house in Auckland with 2 adults and two children respectively. 


\section{Conclusion}

In this study, the effect of five weather variables on electricity consumption was studied using the linear regression analysis and found that temperature and relative humidity showed correlation with the electricity consumption with regression values of 0.438 and 0.335 respectively. A predictive model based on the recurrent neural network consisting of mean temperature as input and electricity consumption as the target variable was tested with three training algorithms, the levenberg-marquadt (LM), bayesian regularization and scaled conjugate gradient. LM back propagation algorithm produced the lowest mean square error of 0.125 as compared to 0.129 and 0.142 for the bayesian regularization and scaled conjugate gradient algorithms respectively. The proposed model was also compared with a benchmark persistence model and it was found that the proposed approach predicted electricity consumption values 24-hours ahead with more accuracy than the persistence approach for a house in Auckland with two adults and two children.

\section{References}

[1] Lukas, G.S., Ugursal, V.I., Modeling of end-use energy consumption in the residential sector: A review of modelling techniques. Renewable and Sustainable Energy Reviews, 13, 1819-1835 (2009)

[2] Flax, B., Intelligent Buildings. IEEE Communications Magazine, 29, 24-27 (1991)

[3] Xia, C., Wang, J., McMenemy, K., Short, medium and long term load forecasting model and virtual load forecaster based on radial basis function neural networks. International Journal of Electrical Power \& Energy Systems, 32, 743-750 (2010)

[4] Maia, C., Goncalves, M., A methodology for short-term electric load forecasting based on specialized recursive digital filters. Computers \& Industrial Engineering, 57, 724-731 (2009)

[5] Niu, D., Gu, Z., Zhang, Y., An AFSA-TSGM based wavelet neural network for power load forecasting. Lecture Notes in Computer Science, 5553, 10341043 (2009)

[6] Tso, G.K.F., Yau, K.K.W., A study of domestic energy usage pattern in Hong Kong. Energy, 28, 1671-1682 (2003)

[7] Al-Garni, A.Z., Zubair, S.M., Nizami, J.S., A regression model for electric energy consumption forecasting in Eastern Saudi Arabia. Energy, 19, 10431049 (1994)

[8] Yan, Y.Y., Climate and residential electricity consumption in Hong Kong. Energy, 23, 17-20 (1998)

[9] Ranjan, M., Jain, V.K., Modelling of electrical energy consumption in Delhi. Energy, 24, 351-361 (1999)

[10] Egelioglu, F., Mohamada, A.A., Guven, H., Economic variables and electricity consumption in Northern Cyprus. Energy, 26, 355-362 (2001) 
[11] Kalogirou, S.A., Bojic, M., Artificial neural networks for the prediction of the energy consumption of a passive solar building. Energy, 25, 479-491 (2000)

[12] Ho, L.K., Hsu, Y.Y., Chen, F.C., Lee, E.T., Liang, C.C., Lai, S.T., Chen, K.K., Short term load forecasting of Taiwan power system using a knowledge-based expert system. IEEE Transactions on Power Systems, 5, 1214-1221 (1990)

[13] Rahman, S., Hazim, O., A generalized knowledge-based short-term loadforecasting technique. IEEE Transactions on Power Systems, 8, 508-514 (1993)

[14] Moghram, I., Rahman, S., Analysis and evaluation of five short-term load forecasting techniques. IEEE Transactions on Power Systems, 4, 1484-1491 (1989)

[15] Khotanzad, A., Afhkhami-Rohani, R., Maratukulam, D., ANNSTLF artificial neural network short-term load forecaster generation three. IEEE Transactions on Neural Networks, 13, 1413-1422 (1998)

[16] Eugen, D., The use of NARX Neural Networks to predict Chaotic Time series. WSEAS Transactions on Computer Research, University of Pitesti, (2008)

[17] Fatih, O.H., Omer, N.G., Mehmet, K., 2008, Hourly solar radiation forecasting using optimal coefficient 2-D linear filters and feed-forward neural network. Solar Energy, 82, 714-726 (2008)

[18] Yadav, A.K., Chandel, S.S., Solar radiation prediction using artificial neural network techniques: A review. Renewable and Sustainable Energy Reviews, 33, 772-781 (2014)

[19] National Institute of Water and Atmospheric Research (http://cliflo.niwa.co.nz/) [cited 10/10/2018] 